\title{
STUDY ON THE DYNAMIC CONSTITUTIVE RELATION OF AZ31B MAGNESIUM ALLOY BASED ON JOHNSON-COOK MODEL
}

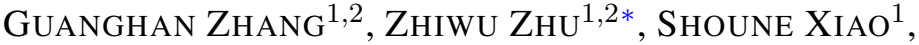 \\ TAO ZHU ${ }^{1}$, QIJUN XIE ${ }^{2}$ \\ ${ }^{1}$ State Key Laboratory of Traction Power, Southwest Jiaotong University, \\ Chengdu, Sichuan 610031, China \\ ${ }^{2}$ Applied Mechanics and Structure Safety Key Laboratory of \\ Sichuan Province, School of Mechanics and Engineering, \\ Southwest Jiaotong University, Chengdu Sichuan 610031, China
}

[Received: 27 February 2018. Accepted: 03 February 2020] doi: 10.7546/JTAM.50.20.01.02

\begin{abstract}
To study the mechanical behavior and constitutive model of a AZ31B magnesium alloy at a high strain rate, the dynamic response of a strain rate ranging from 1630 to $3544 \mathrm{~s}^{-1}$ to the AZ31B magnesium alloy was studied by conducting a split Hopkinson pressure bar experiment. The dynamic stress-strain behavior of the AZ31B magnesium alloy at a high strain rate was analyzed. The results showed that the AZ31B magnesium alloy undergoes a change from an isotropic hardening to a strain softening effect, and no strain rate effect is apparent. In addition, an improved Johnson-Cook constitutive model is employed to consider the influence of damage on the material and determine the parameters for the model. The improved Johnson-Cook model combined with the damage failure model can demonstrate the isotropic hardening behavior of the AZ31B magnesium alloy and the strain softening behavior caused by the damage. The fitting result was determined to be good as compared with the experimental results.
\end{abstract}

KEY WORDS: magnesium alloy; Hopkinson experiment; dynamic mechanical properties; constitutive equation; damage.

\section{INTRODUCTION}

Magnesium is a light metal and its alloys have high specific strength and specific rigidity. In addition, they have excellent damping performance, good dimensional stability, and are widely used in aerospace, weapons, transportation, etc. Magnesium alloy material not only bears the normal design of the load, but also bears the deformation and damage caused by other high strain rate loads such as impact load and explosion [1]. Hence, under high strain rate conditions, the theoretical analysis,

\footnotetext{
${ }^{*}$ Corresponding author e-mail: zzw4455@163.com
} 
and experimental research of the dynamic mechanical behavior of magnesium alloys are urgent problems to be studied. The mechanical properties of AZ31B deformed magnesium alloy are studied, and the constitutive equation is determined. Exploring the deformation mechanism under different conditions not only lays the necessary theoretical foundation for the development of high-strength fine-grained magnesium alloys, but also promotes further application of magnesium alloys in many fields.

Researchers have paid close attention to the theoretical analysis and experimental research of the dynamic mechanical properties of magnesium alloy. Mukai et al. [2] studied the deformation behavior and fracture mechanism of a magnesium alloy under impact loading using a split Hopkinson pressure bar (SHPB) technique. Yokoyama et al. [3-5] observed that the slip mechanism is the main method for plastic deformation in the study of the deformation mechanism of three kinds of magnesium alloys at high strain rates. El-Magd et al. [6] studied the dynamic mechanical properties of the AZ80 magnesium alloy at different loading strain rates through a large number of experiments. The results showed that the fracture sensitivity of magnesium alloys is not immutable and will increase with the increase of strain rate. Koichi Ishikawa et al. [7] studied the dynamic compression properties of solid solution AZ91 magnesium alloys at different temperatures. It was observed that the magnesium alloy exhibits strain hardening, but it soon develops strain softening at low strain rate $\left(10^{-3} \mathrm{~s}^{-1}\right)$ and at a temperature higher than $573 \mathrm{~K}$; The results are surprising at high strain rates $\left(10^{3} \mathrm{~s}^{-1}\right)$ and strain intensification effects occur at all temperatures, especially at room temperature. B. Li et al. [8] studied the dynamic mechanical properties of ZK60 magnesium alloy under equal channel angular pressing. Twins are the main cause of achieving yield. Slip mechanism at high strain rate is the main method for plastic deformation. Gao et al. [9] studied the dynamic compressive stress-strain response and related micro-structural evolution of magnesium alloys by using SHPB. It was observed that the magnesium alloy exhibits metal deformation characteristics of a close-packed hexagonal structure. Sanjari et al. [10] tested the dynamic deformation of magnesium alloys at room temperature using the SHPB device. The results showed that increasing the strain rate (within the quasi-static range) at the same temperature is favorable for dynamic recrystallization. With the increase in the strain rate, the number of twin integrals in the magnesium alloy will continue to increase.

The establishment of constitutive model of magnesium alloy is the major research field of scholars at present. In the study by Xie et al. [11], based on the theory of crystal plasticity and the strain rate sensitivity control method used in the PrestonTonks-Wallace model, a new dynamic constitutive model was established to describe the dynamic stress-strain response of the AZ31B alloy. It was observed that the strain rate almost does not affect the dynamic plastic deformation of the AZ31B alloy under dynamic compression load, and the strain rate effect becomes apparent under 
dynamic tensile load. Xiao et al. [12] studied the compressive dynamic stress-strain response of two representative magnesium alloys, AZ91D and AZ31B, at high strain rates and high temperatures, and the two alloys are well described by the JohnsonCook (J-C) model. However, with the change of strain rate, J-C model does not reflect the strain rate sensitivity. Ahmad [13] studied magnesium alloy AZ31B in different rolling directions with a strain rate in the range of $10^{-4}$ to $3500 \mathrm{~s}^{-1}$. The $\mathrm{J}-\mathrm{C}$ model was used to describe the magnesium alloys under loading at different strain rates. Except for the initial stage, the rest was well fitted. Hou et al. [14] improved the temperature terms in the $\mathrm{J}$ - $\mathrm{C}$ model based on the phenomenological and empirical constitutive models. The new model can be used to describe the $\mathrm{Mg}$ Gd-Y alloy over a wide temperature range with high strain rate. Mao et al. [15] performed a compression experiment at high strain rates. The constitutive equation of the AZ31B magnesium alloy was obtained from the experimental data. It was observed that the alloy has strain rate insensitivity with the increase in strain rate under high loading strain rates. From the above studies, it can be concluded that the theoretical and experimental aspects of the impact of magnesium alloys have been studied in depth. However, the constitutive equation of the magnesium alloy by considering the damage has not been studied yet.

In this study, the dynamic stress-strain curves of the AZ31B magnesium alloy were obtained by the impact experiment under different high loading strain rates by using the SHPB. The mechanical behavior of the AZ31B magnesium alloy under the impact dynamic load was demonstrated. The relationship between the dynamic loading conditions and the mechanical properties of the composites was analyzed, and the classical $\mathrm{J}-\mathrm{C}$ constitutive model was reasonably improved considering the damage. The material parameters of the improved model of the AZ31B magnesium alloy were determined, and the stress-strain curves were obtained via experiments. The curves were compared and the results obtained were good. The rationality of the model improvement was verified.

\section{LABORATORY EQUiPMENT AND PRINCIPLES}

The dynamic compression experiment of the AZ31B magnesium alloy was carried out by using a SHPB system with a cylindrical specimen size of $\phi 10 \times 8 \mathrm{~mm}$. The experimental principle is shown in Fig. 1.

The components of the SHPB system consist of a loading device, bar components, and data acquisition and recording system. Further, the diameters of the striker, incident bar, transmitted bar and absorption bar are $14.5 \mathrm{~mm}$, the length of the incident bar is $400 \mathrm{~mm}$, the length of the transmitted bar is $525 \mathrm{~mm}$. The speed of the striker can be up to $60 \mathrm{~m} / \mathrm{s}$, and the sampling frequency of the super dynamic strain gauge is $1 \mathrm{MHz}$. The resulting waveform data is processed in a two-wave process [16], 
6 Study on the Dynamic Constitutive Relation of AZ31B Magnesium Alloy ...

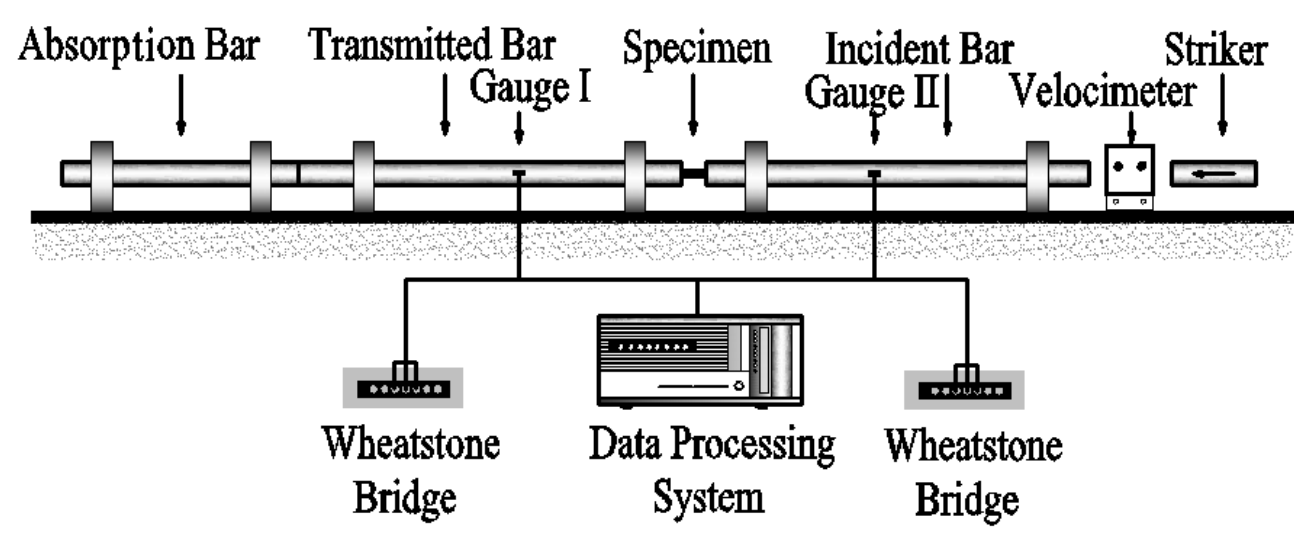

Fig. 1. SHPB schematic.

which is based on one-dimensional and uniform assumptions and is derived from one-dimensional elastic wave propagation theory. The measurement sensor of the SHPB experiment uses a strain gauge attached to the middle of the metal pressure bar. The incident strain wave, reflected strain wave on the incident bar, and transmission strain wave on the transmitted bar are all recorded through the measurement sensor. In this experiment, first, by adjusting the cylinder pressure, the bullets are issued by the pressure inside the cylinder. The speed of the bullet depends on the control of the air pressure, and thus, the control of the load strain rate. The relationship between the cylinder pressure and speed of bullet is shown in Fig. 2.

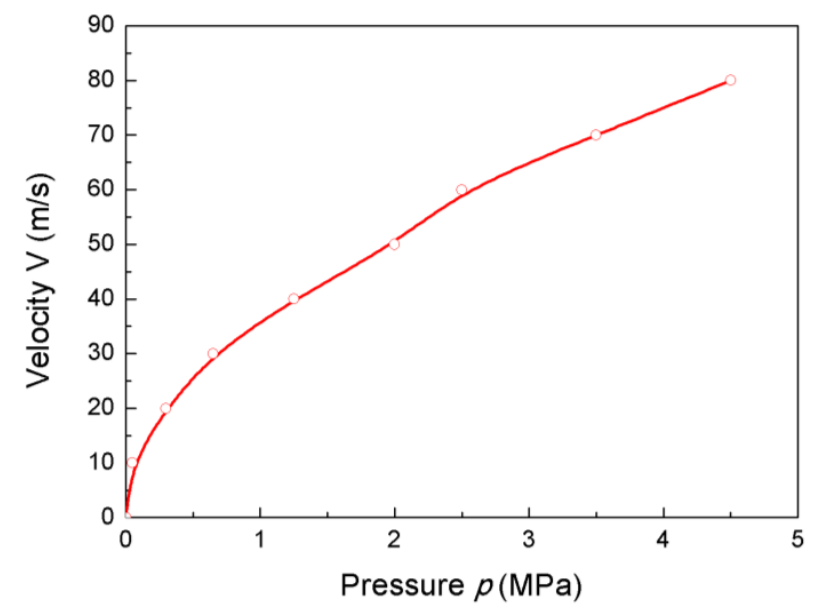

Fig. 2. Relationship between pressure and bullet velocity. 
The relationship between pressure and strain rates, as shown in Table 1, depends on the mechanical properties of the material.

Table 1. Relationship between pressure and strain rates.

\begin{tabular}{lccccc}
\hline \hline Pressure $(\mathrm{MPa})$ & 0.2 & 0.4 & 0.5 & 0.6 & 0.8 \\
\hline Strain rates $\left(\mathrm{s}^{-1}\right)$ & 1630 & 2092 & 2469 & 2950 & 3544 \\
\hline \hline
\end{tabular}

Through the one-dimensional stress wave theory and homogeneity hypothesis [17], the strain rate $\dot{\varepsilon}(t)$, strain $\varepsilon(t)$, and stress $\sigma(t)$ of the material are obtained.

$$
\begin{gathered}
\dot{\varepsilon}(t)=-\frac{2 C_{0}}{l_{s}} \varepsilon_{r}, \\
\varepsilon(t)=-\frac{2 C_{0}}{l_{s}} \int_{0}^{t} \varepsilon_{r} d t, \\
\sigma(t)=\frac{A}{A_{s}} E \varepsilon_{t},
\end{gathered}
$$

where $C_{0}$ is the velocity of the elastic bar, $A$ and $E$ are the cross-sectional area and elastic modulus of the pressure bar, respectively, $l_{s}$ and $A_{s}$ are the length and cross-sectional area of the specimen, respectively, $\varepsilon_{r}$ is the reflected wave, and $\varepsilon_{r}$ is the transmitted wave. The relationship between the stress, strain, and strain rate of the specimen and time is obtained, and subsequently the relationship among stress, strain, and strain rate is obtained.

\section{EXPerimental Process ANd Results}

The load strain rates for the dynamic strain test are $1630 \mathrm{~s}^{-1}, 2092 \mathrm{~s}^{-1}, 2469 \mathrm{~s}^{-1}$, $2950 \mathrm{~s}^{-1}$, and $3544 \mathrm{~s}^{-1}$, and each load strain rate is repeated twice. The repeatability determines whether the third experiment shall be conducted. The compressive stressstrain curve is shown in Fig. 3.

It can be observed from Fig. 3 that the stress-strain curves of the AZ31B magnesium alloy with different strain rates are coincident in the elastic phase and the yield stress tends to be consistent; further, the curve has a high degree of coincidence during the plastic hardening stage. The results show that the AZ31B magnesium alloy does not show apparent strain rate effect at high strain rates. Furthermore, as the stress increases continuously, the material exhibits apparent strain hardening effect under dynamic compression loading; however, over the peak stress, the strain softening behavior begins. It can be observed from the figure that, in the plastic hardening stage, the stress-strain curve is almost linear, indicating that the AZ31B magnesium 
8 Study on the Dynamic Constitutive Relation of AZ31B Magnesium Alloy ...

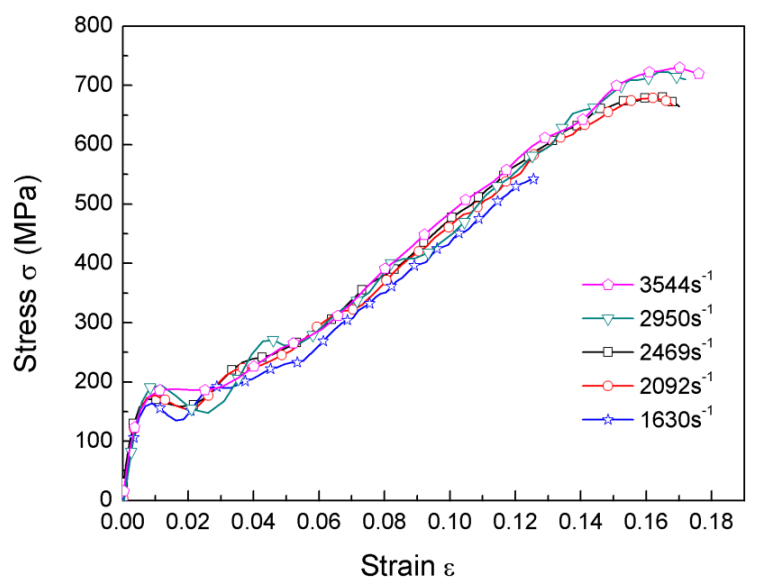

Fig. 3. Stress-strain curve under impact compression loading.

alloy exhibits the characteristics of isotropic hardening under the impact dynamic load.

In order to better explain the dynamic mechanical properties of the AZ31B magnesium alloy, a quasi-static compression experiment of the AZ31B magnesium alloy was carried out by using RPL100 material testing machine. The sample is cylindrical with the dimensions $\phi 10 \times 8 \mathrm{~mm}$. The quasi-static loading strain rates were $0.0001 \mathrm{~s}^{-1}, 0.001 \mathrm{~s}^{-1}, 0.01 \mathrm{~s}^{-1}$, and $0.05 \mathrm{~s}^{-1}$. The experiment was repeated twice for each loading strain rate, and the decision to perform a third experiment depended on the repeatability. The stress-strain curve under quasi-static is shown in Fig. 4.

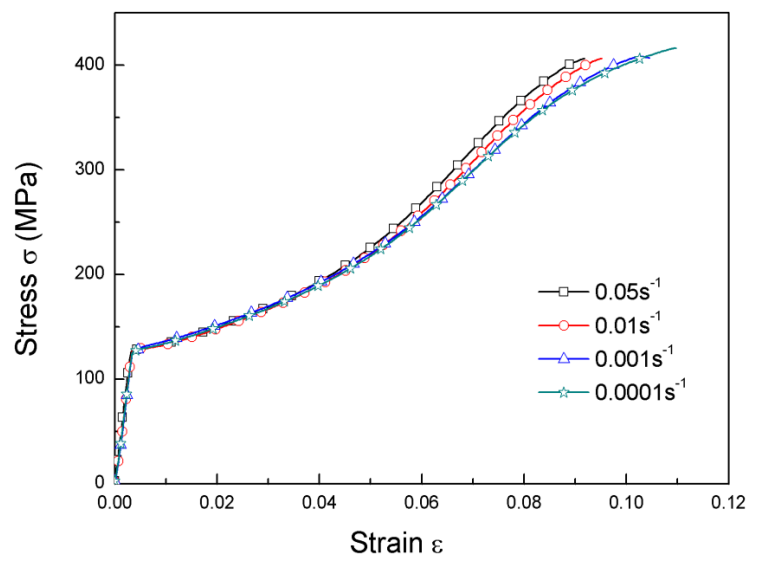

Fig. 4. Stress-strain curve under quasi-static loading. 
It can be observed from Fig. 4 that the material modulus of elasticity undergoes no changes in the loading process, and the yield stress is $130 \mathrm{MPa}$. It can also be observed that the AZ31B magnesium alloy has a very apparent strain hardening phenomenon, i.e., the flow stress in the plastic section significantly increases with the increase in strain, and the maximum stress is higher than the yield stress of $270 \mathrm{MPa}$. The strain rate changes from $0.0001 \mathrm{~s}^{-1}$ to $0.05 \mathrm{~s}^{-1}$, and the yield stress exhibits no apparent changes. It can be observed that the AZ31B magnesium alloy has no strain rate effect at low strain rates. It can also be observed from the figure that the strain hardening phenomenon becomes weaker near the maximum stress because the microcracks and micro-voids inside the magnesium alloy begin to converge and gradually form macroscopic cracks, and the material is finally destroyed.

The quasi-static compression curve of the AZ31B magnesium alloy is compared with the dynamic stress-strain curve, and the difference between the mechanical properties of the AZ31B magnesium alloy at high and low strain rates can be observed more intuitively as shown in Fig. 5.

As evident from Fig. 5, the yield strength of the magnesium alloy under impact loading is slightly higher than that in quasi-static loading; whereas the strain hardening rate under impact loading is significantly higher than that under quasi-static loading. It has been demonstrated that the mechanical properties of the magnesium alloy are different for loading at high and low strain rates. It can also be observed from the figure that, irrespective of whether it is quasi-static or dynamic compression, the magnesium alloy has a large plastic deformation. Slip and twins are the main mechanisms of the plastic deformation of magnesium alloys. According to research

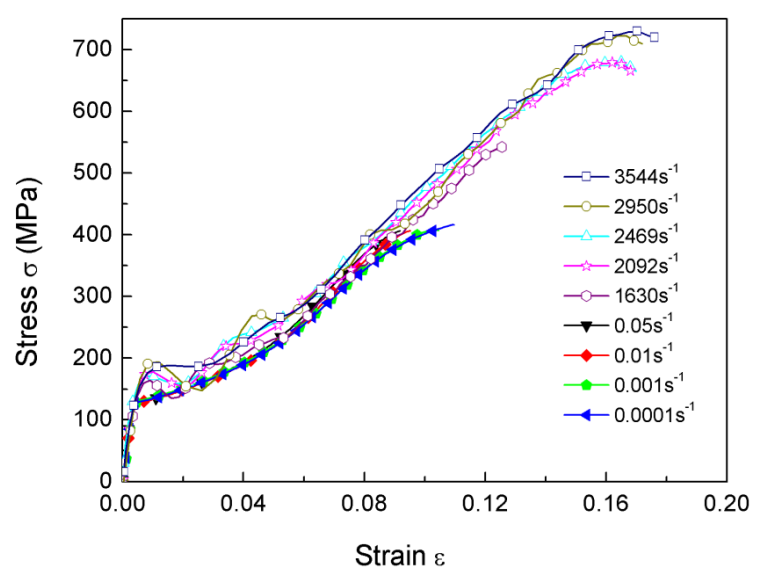

Fig. 5. Comparison between dynamic compression and quasi-static compression stress-strain relationship. 
by Xie [18], the deformation speed of twins is very fast [19], and when the direction of force is favorable for the twins, it will occur preferentially. Therefore, both the external deformation rate and strain rate has little effect on plastic deformation. The relatively slow dislocation slip velocity is attributed to the effect of the strain rate. At the same time, when the direction of force is not conducive to dislocation slip, the occurrence of twins will cause the grain to rotate toward a direction favorable for dislocation slip, which in turn triggers dislocation slip. Under the impact load, the internal dislocation density of the magnesium alloy continues to increase with the increase in deformation. The dislocation adjusts the direction of the slip by rotation under the action of the stress, and then moves along the slip direction. The dislocation slip causes the magnesium alloy to undergo macroscopic plastic deformation. In the initial stage of deformation, the plastic deformation of magnesium alloy is dominated by the twins with fast deformation rate and rate independence. With the increase of strain, the slip mechanism also starts to operate and the contribution to the plastic deformation of magnesium alloy increases, which leads to an increase in the rate sensitivity of the magnesium alloy.

\section{Johnson-Cook Constitutive Model}

Compared with the commonly used constitutive models, the J-C model and the ZerrilliArmstrong model [20] are more frequently used. Since the J-C model is suitable for describing the dynamic behavior of metal materials from low strain rate to high strain rate, it can even be used for quasi-static deformation analysis. This paper studies the J-C model. The results of the data analysis of quasi-static and dynamic experiments of magnesium alloys show that the AZ31B magnesium alloy has apparent strain hardening at the stage of compression and plasticity. Furthermore, the J-C model considers factors such as temperature, strain rate, and strain. The J-C model is simple and has a clear physical explanation. Therefore, the J-C model can describe the dynamic mechanical properties of the AZ31B magnesium alloy. The classical J-C model prototype is represented as [21]:

$$
\sigma=\left(A+B \varepsilon^{n}\right)\left[1+C \ln \left(\frac{\dot{\varepsilon}}{\dot{\varepsilon_{0}}}\right)\right]\left[1-\left(\frac{T-T_{0}}{T_{m}-T_{0}}\right)^{m}\right],
$$

where $A, B, C, n$, and $m$ are undetermined coefficients that are obtained through experiments. Further, $\sigma$ is the rheological stress of the material; $A$ is the elastic yield strength of the material; $B$ is the equivalent plastic strain of the material; $C$ is the strain rate effect coefficient; $n$ is the strain index; $m$ is the coefficient of softening of the material; $\dot{\varepsilon}_{0}$ and $\dot{\varepsilon}$ are the reference strain rate and the equivalent plastic strain rate, respectively; $T$ is the material deformation temperature; $T_{0}$ is the set reference 
temperature; $T_{m}$ is the material melting point temperature. The experimental temperature of the magnesium alloy is always room temperature. However, under the highspeed impact condition, the heat generated by plastic deformation of the material cannot be rapidly diffused to the outside and accumulates, causing the temperature to rise; hence, increasing the adiabatic temperature. According to the literature [18], for AZ31B magnesium alloy, when the temperature is less than $300^{\circ}$, it has little effect on flow stress under the impact compression load. According to calculations, the highest adiabatic temperature rise of AZ31B magnesium alloy in this experiment is $87^{\circ}$, far below $300^{\circ}$. Thus, the adiabatic temperature rise has little effect on the mechanical properties of AZ31B magnesium alloy, and is negligible. In other words, the temperature effect does not have to be considered in the model. Then the J-C model can be written as:

$$
\sigma=\left(A+B \varepsilon^{n}\right)\left[1+C \ln \left(\frac{\dot{\varepsilon}}{\dot{\varepsilon}_{0}}\right)\right] .
$$

First, the reference strain rate is considered as $0.0001 \mathrm{~s}^{-1}$ according to the literature [22]. Since the yield strength of the magnesium alloy is $130 \mathrm{MPa}, \mathrm{A}$ is considered as $130 \mathrm{MPa}$. All the parameters of the AZ31B magnesium alloy under the $\mathrm{J}-\mathrm{C}$ model are calculated, as shown in the Table 2.

Table 2. J-C model parameter values.

\begin{tabular}{ccccc}
\hline \hline$A$ & $B$ & $C$ & $n$ & $\dot{\varepsilon}_{0}$ \\
\hline $130 \mathrm{MPa}$ & $17300 \mathrm{MPa}$ & 0.0021 & 1.74 & $0.0001 \mathrm{~s}^{-1}$ \\
\hline \hline
\end{tabular}

The constitutive equation of the magnesium alloy is obtained as:

$$
\sigma=\left(130+17300 \varepsilon^{1.74}\right)\left[1+0.0021 \ln \left(\frac{\dot{\varepsilon}}{\dot{\varepsilon}_{0}}\right)\right] .
$$

The results are compared with the predicted results. The results are shown in Fig. 6.

It can be observed from Fig. 6 that the J-C model fits better under the strain rate of $1630 \mathrm{~s}^{-1}$, but in the case of the integrated curve, the overall effect is not very satisfactory. The strain hardening effect of the J-C model is too apparent and cannot reflect the strain softening behavior of the AZ31B magnesium Alloy, and therefore, the model must be improved.

\section{IMPROVED JOHNSON-COOK MODEL}

The normal J-C model can only reflect the strain hardening behavior of the material, but the AZ31B magnesium alloy has apparent strain softening in the dynamic 
12 Study on the Dynamic Constitutive Relation of AZ31B Magnesium Alloy ...

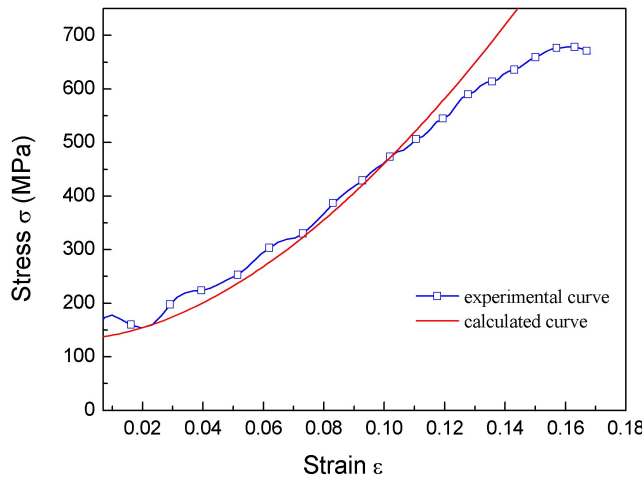

(a) $\dot{\varepsilon}=1630 \mathrm{~s}^{-1}$

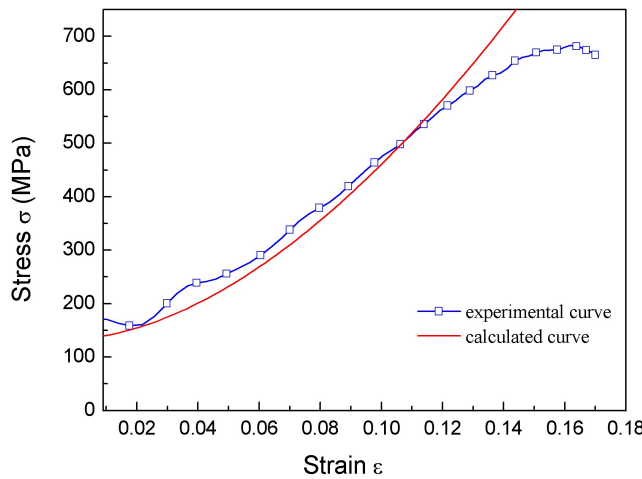

(c) $\dot{\varepsilon}=2469 \mathrm{~s}^{-1}$

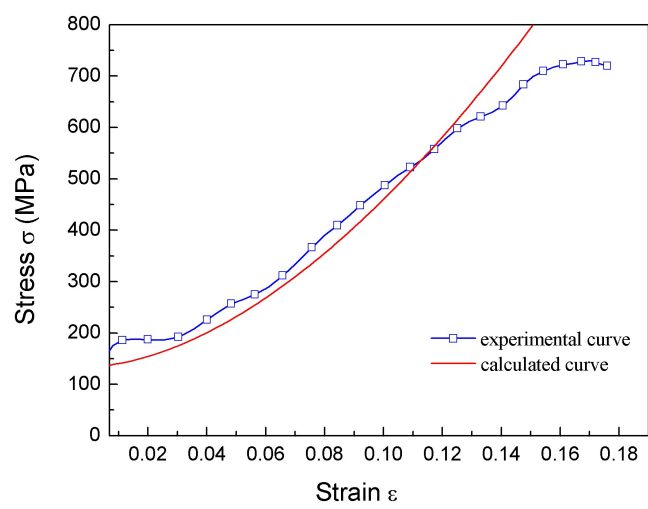

(c) $\dot{\varepsilon}=3544 \mathrm{~s}^{-1}$

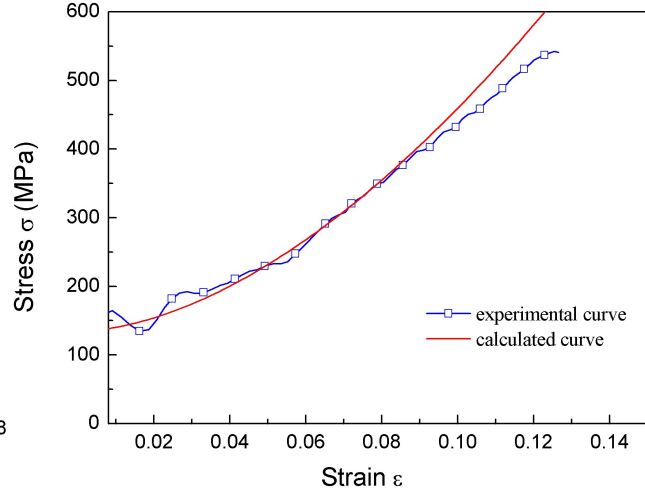

(b) $\dot{\varepsilon}=2092 \mathrm{~s}^{-1}$

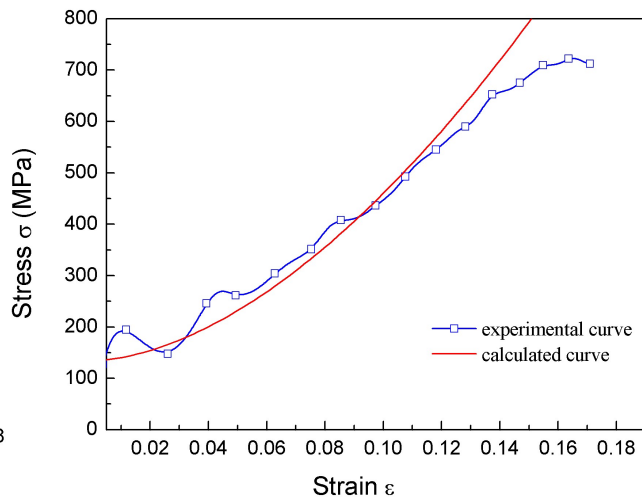

(d) $\dot{\varepsilon}=2950 \mathrm{~s}^{-1}$

Fig. 6. Comparison between the compression test curves and theoretical curves at different strain rates. 
compression experiment, and thus, it is necessary to improve the $\mathrm{J}-\mathrm{C}$ model. The AZ31B magnesium alloy material will inevitably produce micro-cracks in the impact of the dynamic process, which is the main reason for the strain softening behavior of the material [23]. In the loading process, the behavior of the progressive damage of the material owing to the accumulation of micro-cracks and pores can be expressed by the parameter $D$, i.e., the damage parameter. The failure model of the $\mathrm{J}$-C yield function is introduced [24]:

$$
\varepsilon^{f}=\left[D_{1}+D_{2} \exp \left(D_{3} \sigma^{*}\right)\right]\left(1+D_{4} \ln \dot{\varepsilon}^{*}\right)\left(1+D_{5} T^{*}\right),
$$

where $\varepsilon^{f}$ is the failure (plastic) strain, $D_{1}-D_{5}$ are the failure model parameters, $T^{*}=\left(T-T_{0}\right) /\left(T_{m}-T_{0}\right)$ is the dimensionless temperature, $\dot{\varepsilon}^{*}=\dot{\varepsilon} / \dot{\varepsilon}_{0}$ is the dimensionless equivalent plastic strain rate, and $\sigma^{*}$ is the ratio of hydrostatic pressure to equivalent stress.

The parameters of the $\mathrm{J}-\mathrm{C}$ failure model of the AZ31B magnesium alloy were obtained from the literature [25] as $D_{1}=-0.35, D_{2}=0.6025, D_{3}=-0.4537$, and $D_{4}=0.206$. Since the change of temperature is not considered, $T^{*}$ is negligible. The J-C failure model of the AZ31B magnesium alloy at different strain rates can be expressed as:

$$
\varepsilon^{f}=\left[-0.35+0.6025 \exp \left(-0.4537 \sigma^{*}\right)\right]\left[1+0.206 \ln \dot{\varepsilon}^{*}\right] .
$$

According to Bridgman [26], $\sigma^{*}$ can be expressed as:

$$
\sigma^{*}=\frac{\sigma_{m}}{\bar{\sigma}},
$$

where $\sigma_{m}$ is the average of the three principal stresses and $\bar{\sigma}$ is the Von Mises equivalent stress.

It is difficult to determine the exact values of $\sigma_{m}$ and $\bar{\sigma}$ through experiment alone. Herein, the finite element numerical simulation is used, and the dynamic simulation of each sample was simulated using ABAQUS. The grid is divided by C3D8 and the three principal stresses $\sigma_{1}, \sigma_{2}, \sigma_{3}$ are calculated when the specimen is damaged at different loading strain rates [27].

Thus, from the definition, we obtain:

$$
\begin{aligned}
& \sigma_{m}=\frac{\sigma_{1}+\sigma_{2}+\sigma_{3}}{3}, \\
& \bar{\sigma}=\sqrt{\frac{1}{2}\left[\left(\sigma_{1}-\sigma_{2}\right)^{2}+\left(\sigma_{2}-\sigma_{3}\right)^{2}+\left(\sigma_{3}-\sigma_{1}\right)^{2}\right]} .
\end{aligned}
$$

The three principal stress values calculated via the simulation are substituted into equations (10) and (11), and the corresponding $\sigma^{*}$ of the samples under loading at different strain rates is obtained by equation (5) as listed in the Table 3 . 
14 Study on the Dynamic Constitutive Relation of AZ31B Magnesium Alloy ...

Table 3. AZ31B corresponding to different strain rates $\sigma^{*}$.

\begin{tabular}{cccccc}
\hline \hline$\dot{\varepsilon} \mathrm{s}^{-1}$ & 1630 & 2092 & 2469 & 2950 & 3544 \\
\hline$\sigma^{*}$ & 1.1944 & 1.1950 & 1.1952 & 1.1955 & 1.1958 \\
\hline \hline
\end{tabular}

Substituting Table 3 into equation (8), the failure strain $\varepsilon^{f}$ corresponding to each sample under loading at different strain rates was obtained.

Table 4. AZ31B different strain rates corresponding to the failure strain.

\begin{tabular}{cccccc}
\hline \hline$\dot{\varepsilon} \mathrm{s}^{-1}$ & 1630 & 2092 & 2469 & 2950 & 3544 \\
\hline$\varepsilon^{f}$ & 0.1428 & 0.1518 & 0.1530 & 0.1591 & 0.1611 \\
\hline \hline
\end{tabular}

Table 4 shows the relationship between the strain rate and failure strain of the AZ31B magnesium alloy. With the increase in the strain rate, the failure strain gradually increases.

Considering that the stress state and strain rate are varied during the dynamic failure process, the damage to the material is defined by Johnson's damage failure model [28]:

$$
D=\sum \frac{\Delta \bar{\varepsilon}^{p}}{\varepsilon^{f}},
$$

where $\Delta \bar{\varepsilon}^{p}$ is the effective plastic strain increment and $D$ is the damage parameter. When the value of $D$ is less than 1 , the material indicates damage, and when $D$ increases to 1 , the material indicates failure.

Obtained from Table 2, Table 3, and Eq. (12), different strain rates correspond to different failure strains; hence, the cumulative damage corresponding to different strain rates is also different. After the material yields, the strain rate tends to be stable. The effective plastic strain increment can be regarded as a fixed value, that is, the damage $D$ increases uniformly. When the cumulative plastic strain reaches the failure strain, $D$ accumulates to 1 , and the material indicates failure.

It is assumed that the elastic deformation of the magnesium alloy under the uniaxial impact loading is small and can be neglected. According to the studies [29,30], the damage model is introduced into the $\mathrm{J}-\mathrm{C}$ model:

$$
\sigma=\left(A+B \varepsilon^{n}\right)\left[1+C \ln \left(\frac{\dot{\varepsilon}}{\dot{\varepsilon_{0}}}\right)\right](1-D) .
$$

Substituting (12) into (13) in order to obtain the improved J-C model with damage 
as follows:

$$
\sigma=\left(A+B \varepsilon^{n}\right)\left[1+C \ln \left(\frac{\dot{\varepsilon}}{\dot{\varepsilon_{0}}}\right)\right]\left(1-\sum \frac{\Delta \bar{\varepsilon}^{p}}{\varepsilon^{f}}\right),
$$

where $A$ is the elastic yield strength of the material; $B$ is the equivalent plastic strain of the material; $C$ is the strain rate effect coefficient; $n$ is the strain index; $\dot{\varepsilon}_{0}$ and $\dot{\varepsilon}$ are the reference strain and equivalent plastic strain rates, respectively; $\Delta \bar{\varepsilon}^{p}$ is the effective plastic strain increment; and $\varepsilon^{f}$ is the failure (plastic) strain.

The parameter values calculated previously are substituted into Eq. (14), and the parameters are adjusted according to the experimental curve. The parameters of the improved J-C model with the damage are obtained, as shown in Table 5:

Table 5. Parameters of the improved J-C model with damage.

\begin{tabular}{ccccc}
\hline \hline$A$ & $B$ & $C$ & $n$ & $\dot{\varepsilon}_{0}$ \\
\hline $130 \mathrm{MPa}$ & $17300 \mathrm{MPa}$ & 0.0018 & 1.66 & $0.0001 \mathrm{~s}^{-1}$ \\
\hline \hline
\end{tabular}

The comparison between the calculated curve and the experimental curve of the improved J-C model is shown in Fig. 7.

Figure 7 reveals that the obtained experimental results can fit very well by (14). Unlike the original J-C model, the improved J-C model considers damage accumulation and can describe some softening effects. It also describes the complete process of the AZ31B magnesium alloy transition from isotropic hardening to strain softening under impact compression. It can be observed that the improved constitutive model can better describe the dynamic mechanical properties of the AZ31B magnesium alloy under impact loading. 
16 Study on the Dynamic Constitutive Relation of AZ31B Magnesium Alloy ...

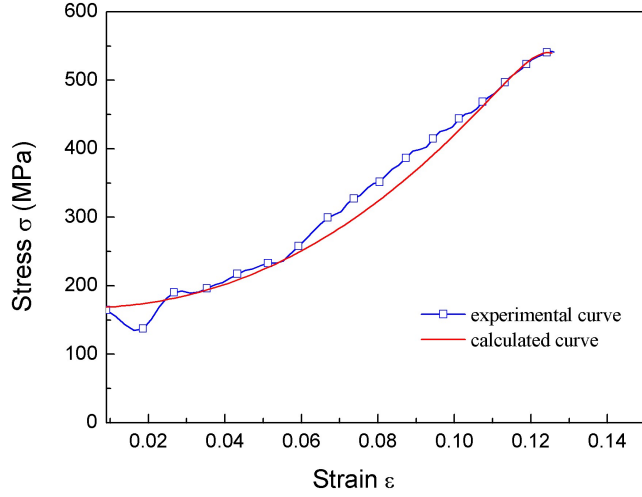

(a) $\dot{\varepsilon}=1630 \mathrm{~s}^{-1}$

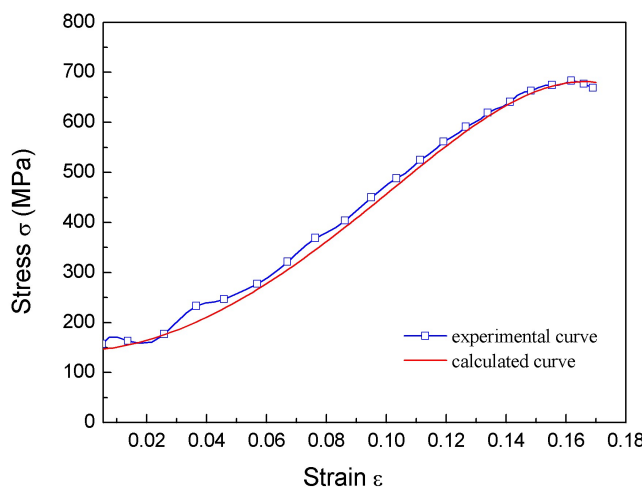

(c) $\dot{\varepsilon}=2469 \mathrm{~s}^{-1}$

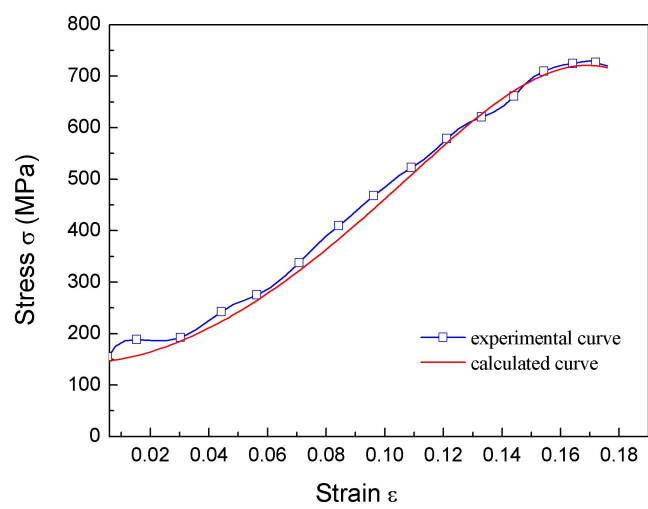

(c) $\dot{\varepsilon}=3544 \mathrm{~s}^{-1}$

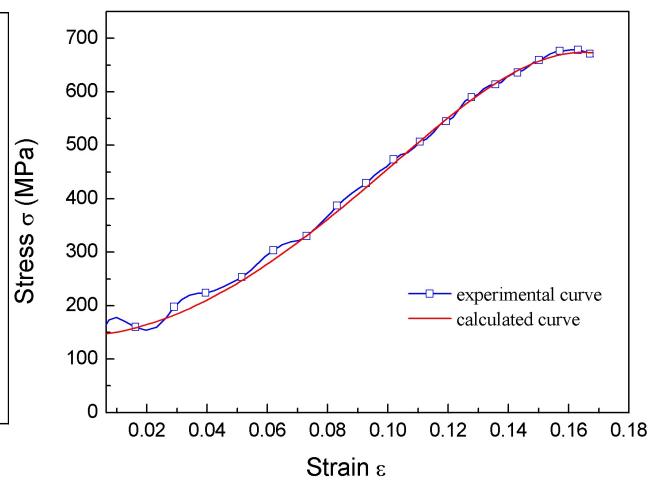

(b) $\dot{\varepsilon}=2092 \mathrm{~s}^{-1}$

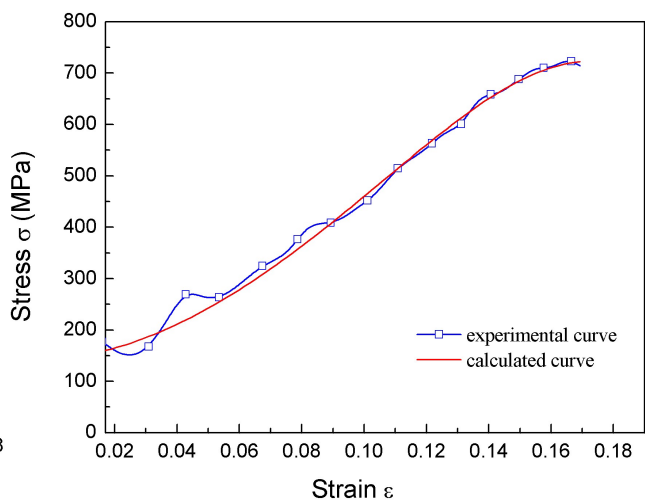

(d) $\dot{\varepsilon}=2950 \mathrm{~s}^{-1}$

Fig. 7. Comparison between the experimental curves and improved $\mathrm{J}-\mathrm{C}$ model curves at different high strain rates. 


\section{CONCLUSion}

In this paper, quasi-static experiments, and dynamic loading experiments of magnesium alloys were carried out to obtain the dynamic stress-strain curves under different loading strain rates. By obtaining a reasonable improvement of the $\mathrm{J}-\mathrm{C}$ model, the dynamic constitutive model of the AZ31B magnesium alloy is obtained. The relevant conclusions are as follows:

1. Through the compression experiment of the AZ31B magnesium alloy under quasi-static and dynamic loading, it can be observed that the flow stress increases with the increase in strain, and the AZ31B magnesium alloy has apparent strain hardening phenomenon. Under dynamic compression load, the strain rate does not affect the dynamic plastic deformation of the AZ31B magnesium alloy, and the alloy has no apparent strain rate effect.

2. The damage is due to the sprout, expansion of the material micro-cracks, and micro-holes at high strain rate. It is observed that damage is the main factor for the change in the mechanical property of the material. Consequently, the strain hardening of AZ31B magnesium alloy is weakened near the peak stress in quasi-static and impact dynamic load, and the strain softening behavior is obvious in the impact dynamic load.

3. The accumulation of micro-cracks and micro-holes result in the softening of the material and gradually destroy the material; consequently, the classic J-C model is no longer applicable to describe the AZ31B magnesium alloy. The $\mathrm{J}-\mathrm{C}$ model is improved by introducing a $\mathrm{J}-\mathrm{C}$ failure model, and the new model can better reflect the dynamic mechanical properties of the AZ31B magnesium alloy, including the strain hardening effect of the AZ31B magnesium alloy and the strain softening caused by damage. The rationality of the improved model is verified through a comparison between the theoretical calculation curve and the experimental curve.

\section{ACKNOWLEDGEMENTS}

This work was supported by the National Key Research and Development Program of China [grant number 2016YFB1200505], the National Natural Science Foundation of China [grant numbers 11672253 and 11972028], and the Opening Foundation of the State Key Laboratory for Strength and Vibration of Mechanical Structures [grant number SV2019_KF-19]. 


\section{REFERENCES}

[1] H. Asgari, J.A. Szpunar, A.G. Odeshi (2014) Texture evolution and dynamic mechanical behavior of cast AZ magnesium alloys under high strain rate compressive loading. Materials \& Design $6126-34$.

[2] T. Mukai, T. Mohri, M. MabUCHI, ET AL. (1998) Experimental study of a structural magnesium alloy with high absorption energy under dynamic loading. Scripta materialia. 39(9) 1249-1253.

[3] T. Yokoyama (2003) Impact tensile stress-strain characteristics of wrought magnesium alloys. Strain 39(4) 167-175.

[4] T. YOKOYAMA (2001) Impact tensile stress-strain characteristics of extrude magnesium alloys. Light Metals 51(10) 544-550.

[5] T. Yokoyama (2003) Tensile and compressive properties of wrought magnesium alloys at high rates of strain. Journal de Physique IV (Proc.). EDP sciences 110 69-74.

[6] E. El-Magd, M. Abouridouane (2006) Characterization, modeling, and simulation of deformation and fracture behavior of the light-weight wrought alloys under high strain rate loading. International Journal of Impact Engineering 32(5) 741-758.

[7] K. Ishikawa, H. Watanabe, T. Mukai (2005) High strain rate deformation behavior of an AZ91 magnesium alloy at elevated temperatures. Materials Letters 59(12) 1511-1515.

[8] B. Li, S. Joshi, K. Azevedo, ET AL. (2009) Dynamic testing at high strain rates of an ultrafine-grained magnesium alloy processed by ECAP. Materials Science and Engineering: A 517(1) 24-29.

[9] C.Y. GAO, L.C. Zhang, W.G. Guo, ET AL. (2014) Dynamic plasticity of AZ31 magnesium alloy: experimental investigation and constitutive modeling. Materials Science and Engineering: A 613(11) 379-389.

[10] M. SAnjari, A. FArzadfar, T. SAKai, ET AL. (2013) A texture and microstructure analysis of high speed rolling of AZ31 using split Hopkinson pressure bar results. Journal of Materials Science 48(19) 6656-6672.

[11] Qijun Xie, Zhiwu Zhu, GuOzheng Kang (2016) Crystal plasticity-based impact dynamic constitutive model of magnesium alloy. International Journal of Mechanical Sciences 119 107-113.

[12] J. Xiao, I.R. Ahmad, D.W. Shu (2014) Dynamic behavior and constitutive modeling of magnesium alloys AZ91D and AZ31B under high strain rate compressive loading. Modern Physics Letters B 28(08) 1450063.

[13] I.R. AhmAD, D.W. Shu (2014) Compressive and constitutive analysis of AZ31B magnesium alloy over a wide range of strain rates. Materials Science and Engineering: A 592 40-49.

[14] Q.Y. Hou, J.T. WANG (2009) A modified Johnson-Cook constitutive model for MgGd-Y alloy extended to a wide range of temperatures. Computational Materials Science 50(1) 147-152. 
[15] Pingli Mao, Zheng Liu, Changyi Wang (2009) Compressive Deformation of AZ31B Magnesium Alloy at High Strain Rate. Chinese Journal of Nonferrous Metals 19(5) 816-820.

[16] H. KOLSKY (1949) An investigation of mechanical properties of materials at very high rates of loading. Proc Phys Soc B62 676-700.

[17] LiLi WANG (2005) "Stress wave basis" [M]. 2nd edition. National Defense Industry Press.

[18] QIJUN XIE (2018) "Experimental and constitutive model study on impact dynamic mechanical behavior of AZ31B magnesium alloy". Chengdu: Southwest Jiaotong University.

[19] J.W. Christian, S. Mahajan (1995) Deformation twinning. Progress in Materials Science 39(1) 1-157.

[20] F.J. ZeriLli, R.W. ARMSTRONG (1987) Dislocation-mechanics-based constitutive relations for material dynamics calculations. Journal of Applied Physics 61(5) 1816-1825.

[21] G.R. Johnson, W.H. CooK (1983) A constitutive model and data for metals subjected to large strains, high strain rates and high temperatures. Proceedings of the 7th International Symposium on Ballistics 21 541-547.

[22] JianXiang Peng (2001) "Study on Constitutive Relation of Tantalum". China Engineering Physics Research Institute.

[23] G.R. Johnson, W.H. Cook (1985) Fracture Characteristics of Three Metals Subjected to Various Strains, Strain Rates, Temperatures and Pressures. Engineering Fracture Mechanics 21(1) 31-48.

[24] V. OBorin, M. BANNiKov, O. NAIMARK, ET AL. (2015) Multiscale study of fracture in aluminum-magnesium alloy under fatigue and dynamic loading. Frattura ed Integrita Strutturale, (34).

[25] Fei Feng, Shangyu Huang, Zhenghua Meng (2014) A constitutive and fracture model for AZ31B magnesium alloy in the tensile state. Materials Science \& Engineering A 594 334-343.

[26] P.W. BRIDGMAN (1952) Studies in large plastic flow and fracture with special emphasis on the effects of hydrostatic pressure. Science 115(2990) 424.

[27] FeI Feng (2014) "Study on High Temperature Deformation Behavior and Mechanism of Magnesium Alloy". Wuhan University of Technology.

[28] G.R. Johnson, W.H. CoOK (1985) Engineering Fracture Mechanics 21 31-48.

[29] Mohanraj Murugesan, Dong Won Jung (2019) Johnson Cook Material and Failure Model Parameters Estimation of AISI-1045 Medium Carbon Steel for Metal Forming Applications. Materials 12(4) 609.

[30] A. Banerjee, S. Dhar, S. Acharyya, D. Datta, N. Nayak (2015) Determination of Johnson cook material and failure model constants and numerical modelling of Charpy impact test of armour steel. Materials Science and Engineering: A 640 200-209. 